\section{Revue d'ethnoécologie}

$20 \mid 2021$

Un siècle d'ethno-machins : le centenaire de la Revue de Botanique Appliquée

\title{
Le teck, Tectona grandis L.f., chez les Arabo- Musulmans du Moyen-Âge
}

Teak, Tectona grandis L.f., among the medieval Arab-Muslims

\section{Françoise Aubaile-Sallenave}

\section{OpenEdition}

\section{Journals}

Édition électronique

URL : https://journals.openedition.org/ethnoecologie/8651

DOI : 10.4000/ethnoecologie.8651

ISSN : 2267-2419

Éditeur

Laboratoire Éco-anthropologie

Référence électronique

Françoise Aubaile-Sallenave, «Le teck, Tectona grandis L.f., chez les Arabo-Musulmans du MoyenÂge », Revue d'ethnoécologie [En ligne], 20 | 2021, mis en ligne le 31 décembre 2021, consulté le 06 mars 2022. URL : http://journals.openedition.org/ethnoecologie/8651 ; DOI : https://doi.org/10.4000/ ethnoecologie.8651

Ce document a été généré automatiquement le 6 mars 2022

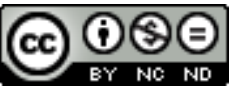

Revue d'ethnoécologie est mis à disposition selon les termes de la licence Creative Commons Attribution - Pas d'Utilisation Commerciale - Pas de Modification 4.0 International. 


\title{
Le teck, Tectona grandis L.f., chez les Arabo-Musulmans du Moyen-Âge
}

Teak, Tectona grandis L.f., among the medieval Arab-Muslims

\author{
Françoise Aubaile-Sallenave
}

\section{Introduction : un parcours scientifique}

1 Arrivée relativement tardivement au laboratoire d'ethnobotanique et d'ethnozoologie du Muséum, j'ai pu y formaliser une démarche personnelle de l'ethnobotanique, dans le droit fil de mes études à la FRA ${ }^{1}$ au Musée de l'Homme auprès d'André Leroi-Gourhan, puis au CEDRASEMI ${ }^{2}$ fondé par Georges Condominas, avec Lucien Bernot et AndréGeorges Haudricourt, tous proches et liés à Roland Portères du laboratoire du Muséum. Mon approche de l'ethnobotanique associe la technologie, l'ethnolinguistique et l'histoire. J'ai voulu en témoigner à l'occasion du centenaire de ce support de notre discipline, la Revue de botanique appliquée (où s'inscrit dorénavant l'ethnoécologie), en introduisant brièvement mon parcours, puis à travers un texte consacré à un arbre majeur de l'Asie du Sud-Est, le teck (Tectona grandis L.f., une plante de la famille des Lamiacées, ex Verbénacées).

2 Fille d'un père marin et forestier, je me suis, depuis mon enfance, intéressée aux bateaux et à la navigation, et un peu plus tard à la technologie marine quand mon père nous a construit un Vaurien, petit dériveur sur lequel j'ai appris à naviguer, avant de monter sur un bateau plus grand, toujours construit par mon père. Avec mes études d'ethnologie, j'ai compris combien les usages des bateaux sont importants. Ma première étude académique a été mon texte de maîtrise sous la direction de A. LeroiGourhan, après une mission sur le terrain, sur les Barcos moliceiros de la Laguna de Aveiro (Portugal), beaux et grands bateaux à fond plat, décorés de saints protecteurs. Ces bateaux approvisionnent en engrais les producteurs de maïs de l'intérieur. Ces engrais sont les algues moliços que ces moliceiros vont chercher dans cette lagune d'eau salée (Aubaile-Sallenave 1969). Puis, mon lieu de naissance, Hanoï, et mes parents - sans y penser et tout naturellement - m'ont orientée vers l'Indochine, où ils ont vécu et où 
mon père a travaillé après sa sortie de l'École forestière de Nancy, après des études à l'Institut agronomique de Paris. C'est ainsi que je me suis tournée vers l'étude des matériaux de construction des bateaux d'Indochine, dont l'ouvrage de Jean B. Piétri (1943) donne une bonne idée de la très grande variété. C'est à ce moment-là que je me suis intéressée à leur technologie bien particulière. Cela va des bateaux entièrement en bois, mais à coque souple, à certains bateaux des centre et sud-Viêtnam dont la coque est en vannerie, très souple également. J'ai entrepris alors ma thèse de $3^{\mathrm{e}}$ cycle sous la direction du professeur Georges Condominas, thèse publiée plus tard en 1987, sous le titre de Bois et Bateaux du Viêtnam. On l'a compris, l'autre aspect intéressant des bateaux vietnamiens et d'Indochine, ce sont les nombreux matériaux végétaux qu'offre le milieu et la technologie navale bien particulière de ces pays qui permet d'utiliser au mieux leurs propriétés. La botanique m'intéressait aussi depuis longtemps et cela m'intéresse toujours, bien qu'au début de ma thèse, je n'aie pas pu faire de terrain en Indochine, alors bouleversée par la guerre. C'est sur documents que j'ai construit cette étude. Celle-ci achevée, je me suis tournée vers une autre grande civilisation, que j'avais étudiée au lycée, de la classe de $3^{\mathrm{e}}$ au baccalauréat et au-delà, grâce à une professeure d'espagnol, Madame Garrigue, qui nous avait fait connaître la civilisation arabo-musulmane médiévale présente pendant sept siècles en Espagne et plus particulièrement en Andalousie. C'est ainsi qu'après ma thèse, intégrée au Museum, j'ai étudié les nombreux apports de plantes, leurs routes et leurs usages, que ces AraboMusulmans nous ont fait connaitre. Plusieurs de mes travaux d'ethnobotanique historique ont d'ailleurs été publiés dans le JATBA ${ }^{3}$.

Beaucoup plus récemment, j'ai pu faire le lien entre l'Asie du sud-est de mes débuts, et l'Amérique latine, à travers l'ethnobotanique des arbres connus, nommés et utilisés par les Hmong immigrés en Guyane, mais originaires du Laos.

\section{Le teck des Arabo-Musulmans}

Le teck, sāj des Arabes, après avoir été utilisé comme bois précieux dans les constructions marines, les monuments prestigieux, trouve aujourd'hui des usages beaucoup plus ordinaires comme meubles de jardin, tables, chaises, chaises-longues, etc. ${ }^{4}$. Les constructions marines n'y recourent plus guère, sinon pour les ponts de luxueux bateaux de plaisance. Mais il a une longue histoire, peut-être encore plus longue que celle que je vais raconter, car il est connu historiquement depuis que les Chinois $^{5}$, les Persans, puis les Arabes, avant même l'Islam, ont commercé les uns avec leur Occident, les autres avec notre Extrême-Orient, au vi siècle et probablement bien auparavant. C'est ce que note Tadeusz Lewicki (1935). C'est surtout le Périple de la mer Erythrée (daté vraisemblablement du $1^{\mathrm{er}}$ siècle A.D.) qui reporte bien plus loin dans le passé le commerce de ce bois depuis Barygaza, ville de la côte nord de l'Inde occidentale ${ }^{6}$ proche des forêts de teck (Casson 1989 : 72 et 73).

5 Le teck était connu depuis longtemps en Inde, dans les massifs montagneux, monts du nord-est jouxtant la Birmanie, mais aussi les Ghatts des côtes occidentales du Konkan ${ }^{7}$ et du Malayalam, côte du Malabar. Ces zones étant les plus accessibles aux Persans puis aux Arabes de la péninsule Arabique (Figure 1). 
Figure 1 : Carte de situation

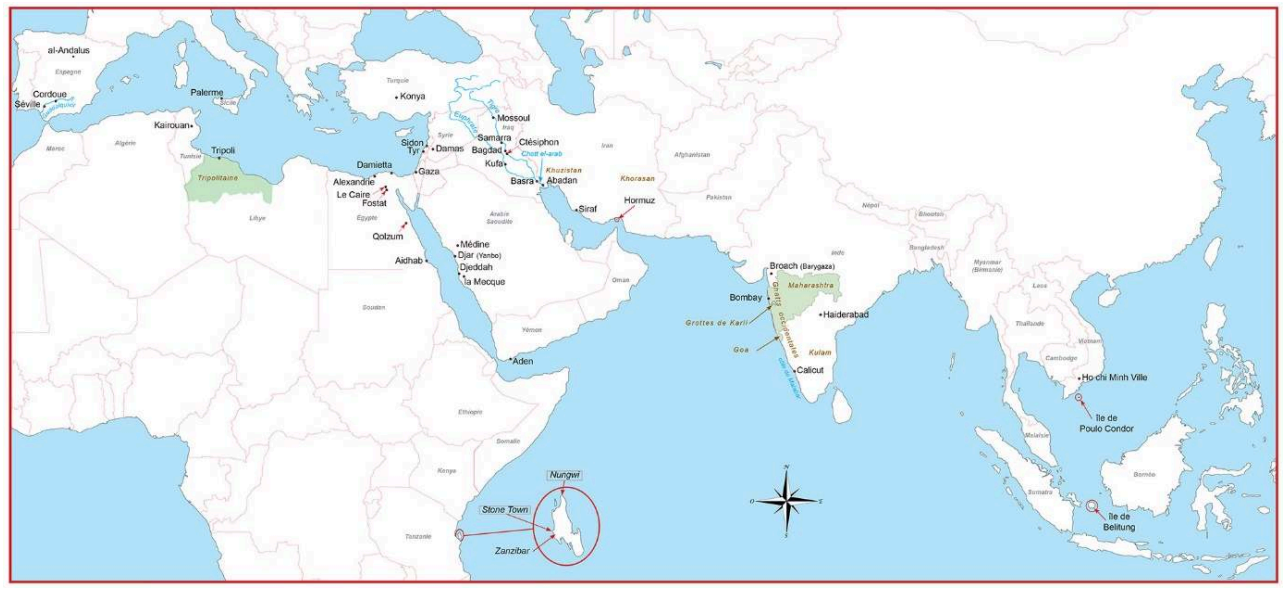

L. Venot

6 Nous connaissons aujourd'hui trois espèces de Tectona de la famille des Lamiacées. Le plus important est Tectona grandis L.f. Il devient un grand arbre, de 25 à $30 \mathrm{~m}$ de fût et $30 \mathrm{~cm}$ de diamètre en moyenne, mais souvent plus. Le tronc est fréquemment cannelé et irrégulier, mais droit (Figure 2). Son aire naturelle dans la péninsule indochinoise est assez restreinte; les noyaux cristallins semblent lui être particulièrement favorables. Elle se limite au bassin supérieur du Mékong, en amont de Pak Lay, au Nord-Laos, en Malaisie, et au nord-est de l'Inde. C'est aujourd'hui l'espèce la plus commune, dite «teck d'Indochine », elle est originaire d'Inde, de Malaisie et de Thaïlande. Elle est cultivée dans toutes les zones tropicales et sub-tropicales. 
Figure 2 : Le teck représenté dans le Hortus malabaricus de H. van Rheede en 1682

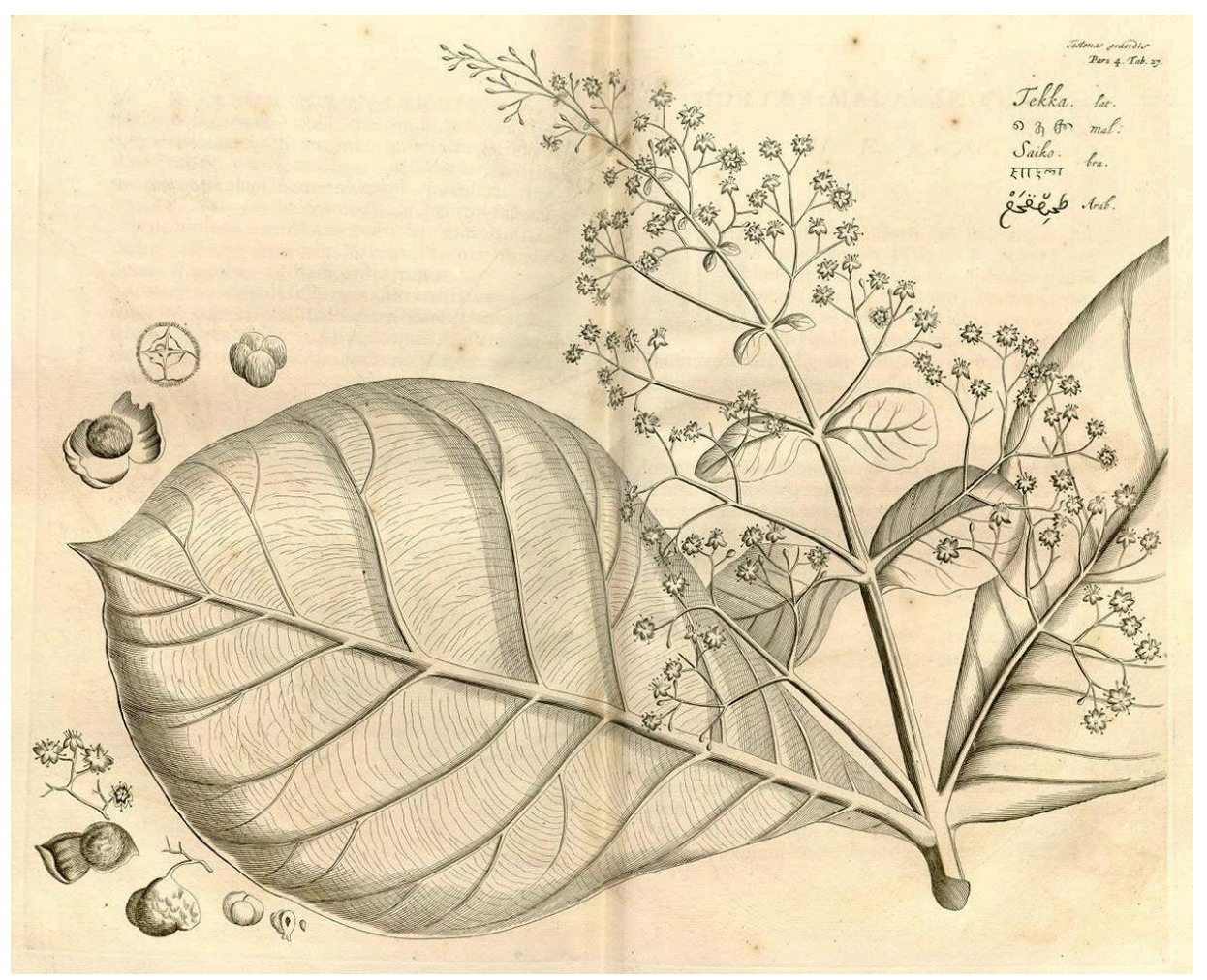

https://www.biodiversitylibrary.org/

7 Une des espèces, le « teck dahat » T. hamiltoniana Wall., est endémique de Birmanie, elle est considérée comme la meilleure des Tectona et elle est donc très menacée. Une troisième espèce, endémique des Philippines, T. philippinensis Benth. \& Hook.f., est également très menacée.

Cet arbre ne poussait spontanément ni au Viêtnam, ni au Cambodge, mais il a été planté par petits bouquets à peu près partout en Indochine. Avant les guerres du début du $\mathrm{Xx}^{\mathrm{e}}$ siècle, on le trouvait en peuplements au Cambodge et au Sud-Vietnam (Anonyme, BEI, 1935). Son aire d'extension est beaucoup plus vaste, jusqu'à Java où les missionnaires bouddhistes l'ont importé d'Inde au début de notre ère (Robequain : 71).

( résiste bien aux insectes et aux intempéries : c'est le meilleur bois de construction navale. Mi-dur, mi-lourd (densité de 0,65 à 0,75 ), il est facile à travailler, mais sa résistance mécanique aux chocs est assez médiocre. Son retrait est très faible au séchage ; il est imputrescible. Il résiste bien aux insectes et assez bien aux tarets marins (Teredo sp. et Bankia sp.). Mais ce qui le fait surtout apprécier des charpentiers de marine, c'est qu'il ne ronge pas les chevilles métalliques, bien au contraire, il les conserve, grâce à l'oléorésine qu'il contient - et cela tout à l'inverse des nombreux bois qui ne protègent pas le fer de l'humidité.

Le teck possède en outre d'excellentes propriétés physiques : il sèche sans se déformer, reste inerte sous les variations d'humidité et il est presque imperméable à l'eau'

Des poutres de teck datant du milieu du vi ${ }^{\mathrm{e}}$ siècle, sont encore en place, dans les murs du grand palais des rois sassanides à Ctésiphon ${ }^{9}$ (Birdwood, First Letter Book, Intro. XXIX, cité par Yule \& Burnell 1984 : 910). 


\section{Le teck dans les constructions navales ${ }^{10}$} importantes et soignées, car il était et reste toujours très cher. Dans tous ses pays d'origine, montagnes de Thaïlande, du Laos et de Birmanie, les forêts appartenaient au souverain. Rangoon, ancienne capitale de Birmanie et centre économique, a dû sa richesse aux constructions de bateaux en teck. Les chantiers navals y étaient installés car on ne pouvait pas exporter ce bois. Dans ses pays d'origine, il est vénéré comme l'arbre-roi (Sallenave 1949 : 34).

Son exploitation, dans les montagnes du nord de la péninsule indochinoise, était assez particulière. L'est-elle encore? En effet les tecks étaient tués par ceinturage ${ }^{11}$, ils mouraient sur pied et n'étaient abattus qu'un ou deux ans plus tard; ils étaient alors bien secs et pouvaient être lancés sans flotteur sur la rivière qui les amenait au lieu de débit (Dauphinot $1905: 629$ ).

C'est lors de ma lecture de l'ouvrage fameux de Mas' ūdī, bağdādī (habitant de Bagdād) du $\mathrm{x}^{\mathrm{e}}$ siècle (c. 896-956), Les prairies d'or, que mon attention a été éveillée. En effet, cet écrivain connaissait bien le teck et les différences entre les constructions navales de l'Océan indien et celles de la Méditerranée :

«On a trouvé dans la Méditerranée, du côté de l'île de Crète, des planches de bois de teck, percées de trous et reliées ensemble par des attaches faites de fibres de cocotier; elles provenaient de vaisseaux naufragés et avaient été le jouet des vagues. Or ce genre de structure n'est en usage que dans l'océan indien. Les vaisseaux qui naviguent en Méditerranée et ceux des Arabes sont tous pourvus de clous, tandis que dans l'Océan indien, les clous de fer ne tiennent pas, parce que l'eau les ronge, les faits fondre et les rend très faibles. Ce qui force les constructeurs à les remplacer pour joindre les planches et bordés, par des fibres de cocotier enduites de graisse et de goudron. ${ }^{12}$ » (al-Mas' ūdī 1961-1995, I : 147).

C'est ce texte qui m'a, il y a pas mal d'années, intrigué sur leur connaissance du bois de teck. Ce sont probablement les Persans, grands navigateurs et connaissant depuis longtemps les productions végétales de l'Inde (riz, aubergine, épinard, safran, gingembre, canne à sucre et autres), et de Chine également (les aromates), qui les ont faites connaître aux Arabes qui, à leur tour, nous les ont faites connaître. Il en va ainsi du teck.

Par ailleurs, ce texte appelle deux remarques. D'abord ces planches venaient d'un bateau qui n'avait probablement pas été construit dans l'Océan Indien, mais plutôt par des constructeurs indiens ou sudarabiques que l'on faisait venir dans les arsenaux de la rive orientale de la Méditerranée et qui ne connaissaient que cette technique des bateaux cousus. Ensuite, les constructeurs n'avaient pas observé les propriétés de ce bois, qui sont de conserver le fer, car ils ont continué à lier les bordés ou bien les planches du pont, avec les fibres entourant les noix de coco, technique de construction navale très ancienne dans l'Océan Indien.

17 Al-Hamadānī, géographe voyageur, détaille au $\mathrm{x}^{\mathrm{e}}$ siècle :

« Allāh a attribué aux pays de Sind et de l'Inde, les diverses sortes de parfums et de joyaux [...], le rhinocéros, l'éléphant, le paon, les bois odoriférants, les encens, l'ambre, le girofle, le galanga [...], le bambou [...], le bois de santal, le teck, le poivre et nombre de choses étonnantes » $(1973: 303)$. 

Méditerranée occidentale. Passant par le Maghreb, ils arrivent et colonisent, dès 711, ce qu'ils nommeront al-Andalūs, l'Andalousie aujourd'hui et beaucoup plus. Ils resteront en Espagne jusqu'en 1609-1611. Ce sont les Aghlabides d'Ifrīqìya, le Maghreb aujourd'hui, qui coloniseront aussi la Sicile à partir de 827. Dans ces deux régions, ils développeront des cultures prospères, prestigieuses et pluri-confessionelles : musulmans, juifs et chrétiens dans al-Andalūs, musulmans et chrétiens en Sicile et partout multilingues. La Sicile est conquise par les Normands chrétiens en 1060 qui, éblouis par cette culture, conserveront et développeront sa prospérité, selon des termes très originaux, pendant plusieurs siècles.

Par leur situation nouvelle, les Arabes du Proche Orient sont devenus marins avec une grande activité navale, commerciale et militaire contre les infidèles, activité soutenue par des arsenaux (« arsenal » est d'ailleurs un terme d'origine arabe dār as-sinā $\bar{a}^{\star} a$ ou dār as-san'ā " maison de la fabrication ", qui a donné l'espagnol atarazana). Ils créent, dans la structure des ports, des arsenaux et des lieux de remise en état de leur flotte. Ce sont, à l'est de la Méditerranée, les arsenaux des côtes syro-palestiniennes et égyptiennes où ils font venir des constructeurs de bateaux sud-arabiques et indiens. À l'ouest, ces arsenaux sont en amont de Séville ${ }^{13}$ et à Palerme, dans le quartier arabe, la Halisa, la Kalsa d'aujourd'hui, au bord de la mer. Les Arabes d'al-Andalūs commerçent avec toute la Méditerranée (Levi-Provençal 1969 : 74).

L'Égypte fatimide (969-1171), ne trouvant plus, malgré ses efforts, suffisamment de bois locaux, à savoir les Fabacées sant (Acacia nilotica) et lebekh (Albizzia lebekh) pour ses constructions marines, fait venir le bois de teck d'Inde occidentale, notamment en 975, 976 et en 995 (Lombard 1972: 130 et Lev 1984, cités in Planhol 2000 : 39). Elle crée également en Tripolitaine plusieurs arsenaux très actifs sur la côte méditerranéenne. Tous utilisent le teck à plusieurs titres. En effet, les forêts des côtes occidentales de l'Inde « fournissaient en bois de teck, quasi imputrescible, toutes les marines de l'océan Indien occidental, du golfe Persique et de la mer Rouge ; les boutres arabes de l'Oman et du Yémen, les navires de Sīrāf et de Bassora, comme ceux de la mer Rouge à Qolzum et Aidhāb, sur la côte égyptienne, et à Djeddah et Djar (aujourd'hui Yanbo) sur la côte de la péninsule Arabique, étaient construits en bois de teck ». (Lombard 1972 : 133). Et par Qolzum, qui est à l'entrée du canal vers le Caire ${ }^{14}$, les bois de l'Inde, dont le teck, arrivaient aux chantiers de Fosțāt, en Égypte, qui l'employaient pour les pièces maitresses des coques (Lombard 1972 : 133).

21 C'est au IX ${ }^{\mathrm{e}}$ siècle que les Arabes d'Iraq, qui, à la suite des Persans, commerçaient déjà avec l'Inde et ce qui est aujourd'hui l'Indonésie, allèrent, probablement portés par la mousson du sud-ouest ${ }^{15}$, jusqu'en Chine, où ils découvrent le thé, dont la recette est donnée dans l'ouvrage du marchand Sulaīmān, daté de 851 (Aubaile-Sallenave 2005 : 158).

Nous avons quelques informations sur les constructions navales des Arabes médiévaux.

Marco Polo (1254-1324), décrit assez précisément les bateaux cousus, qu'il voit à Hormuz, faits en bois de cocotiers; il parle aussi de l'huile de poisson qui sert de calfatage, mais ne nomme pas le teck (Yule 1903: 108, 126). Bateaux cousus dont Ya'aqūbī († après 905), nous dit qu'ils sont construits à Ubūlla, faubourg de Basra et qu'ils voguent jusqu'en Chine (1937 : 226).

Revue d'ethnoécologie, 20 | 2021 
24 C'est le travail des archéologues sur le naufrage d'un bateau, soit arabe, soit indien, au IX siècle près de Belitung, (une île entre Sumatra et Bornéo), qui nous permet aujourd'hui de connaître les bois employés. Ils ont pu être identifiés, malgré les difficultés liées à la disparition de leur cellulose : l'ensemble de ces bois montre que ce navire était d'une construction très soignée et précise pour utiliser au mieux les propriétés de chacun d'eux. C'est un bateau dont les bordés étaient cousus, et donc construit par des Indiens ou des Arabo-Persans de la mer de l'Inde :

«Ce sont le bois de rose, Dalbergia sp ou Pterocarpus sp des Fabacées pour le poste arrière, le teck Tectona grandis pour les barreaux de pont, un cyprès, Cupressus sp des Pinacées pour les membrures du plafond et puis Artocarpus sp des Moracées, pour une boite trouvée dans le naufrage. La charpente et le manche de l'ancre, étaient soit d'Afzelia, une Fabacée, soit d'Amoora sp, une Méliacée. Les bordés de la coque semblent être d'Amoora sp et la cale de la quille est probablement de Ficus sp., une Moracée. » (Flecker 2000 : 13, ma traduction).

« De tous ces bois, seul Afzelia est africain » (Flecker 2000, idem). Les autres sont, dans leur majorité, indiens.

Ce bateau venait de Chine et transportait un bel et grand ensemble de poteries chinoises ${ }^{16}$ qui ont permis de dater à la fois le bateau et le naufrage : ils sont du $\mathrm{Ix}^{\mathrm{e}}$ siècle.

Aujourd'hui, on observe à Zanzibar ces embarcations millénaires que sont les dhow, toujours en usage ${ }^{17}$. Ces bateaux voguent à voile, simple ou multiples. Ils ont une stabilité, une robustesse et une rapidité reconnues (Yajima 1977, Vosmer 1977a ; Ministry of Informa-tion, Sultanate of Oman 2012). Nungwi et Stone Town sont les lieux de construction de ces embarcations où l'on se sert exclusivement de ferrures manuelles récupérées. Les activités de pêche de l'archipel sont principalement faites avec ces dhow, qui font aussi du cabotage pour transporter des marchandises et des passagers. Ces communautés littorales de pêcheurs ont une organisation sociale et des activités économiques, s'appuyant en grande partie sur la construction et l'utilisation de ces embarcations. Mais la rareté des bois exotiques (acajou, teck, manguier) utilisés dans leur construction, condamnent ces dhow traditionnels à disparaître. (Figure 2) (Hawkins 1981 : 20-21).

\section{Le teck dans les mosquées et monuments religieux}

L'autre usage important du teck a coïncidé avec les nouvelles constructions qu'exigeaient les débuts de l'Islam (né en 622), les mosquées du Moyen Orient. Le teck, importé de l'Inde occidentale, servait à couvrir, c'est-à-dire à faire le soubassement ou la charpente des toitures. La Katbah, dans la Grande Mosquée de la Mecque, le lieu le plus sacré de l'Islam, construite en marbre et mosaïque, est ainsi couverte de bois de teck (Terrasse 1971 :166). Ibn Ğubayr, au XII ${ }^{\mathrm{e}}$ siècle, note que ce plafond (charpente) est soutenu par trois piliers de bois de teck fort hauts (Ibn Jobaïr 1951 : 95). Ibn Bațtūta, un siècle plus tard et citant son prédécesseur, a été lui aussi impressionné par "ces colonnes excessivement élevées en bois de teck et qui sont séparées l'une de l'autre de la distance de quatre pas; elles occupent le milieu de l'espace qui constitue l'intérieur de l'illustre Ka'bah. » (Ibn Bațțūta 1982, $1: 310)$. dans toute sa longueur, à Bașra, jusqu'au $x^{\mathrm{e}}$ siècle et presque essentiellement pour la 
construction navale et les constructions prestigieuses qu'étaient les mosquées (Lombard $1971: 175$ ).

30 À Médine, le calife 'Uțmān (574-656) agrandit et embellit la Mosquée construite par le Prophète. Il y dresse des piliers de pierres, renforcés par des colonnes de fer et de plomb; il la recouvre de bois de teck, sāj, et y construisit un mihrā $b^{18}$ qu'admire encore ibn Batțūta au XIV siècle (Ibn Bațțūta 1982 I :271). La mosquée avait été agrandie à plusieurs reprises, la dernière, décrite par Ibn Bațțūta, fut réalisée par « el-Walīd, $6^{\mathrm{e}}$ calife omeyade (668-705-715) fils d'abd el-Malik (646-705), $5^{\text {e }}$ calife [...] et ce fut 'Omar, $8^{e}$ calife (682-720 à Alep) fils d'abd el-Azīz qui dirigea les travaux. El-Walīd, l'augmenta, l'embellit et en accrut beaucoup la solidité. Il la construisit de marbre et de bois de teck doré », nous dit Ibn Bațtūita (op. cit. : 271).

Al-Hamadānī (début du $\mathrm{x}^{\mathrm{e}} \mathrm{s}$.), géographe et historien persan, décrit les débuts de la mosquée de Médine, la mosquée du prophète. «La mosquée fut construite à l'époque du prophète... sa toiture était faite de ğarìd (branches de palmier sans feuilles), ses colonnes étaient de bois de palmier ; 'Umar (644) l'agrandit, puis 'Uțmān (656) ${ }^{19}$ la modifia, il y mit des colonnes de pierres taillées et un toit de teck... Actuellement (c'est al-Hamadānī qui l'écrit en 903), la mosquée à double toiture [...] et le miḥrāb et la maqșūra ${ }^{20}$ sont en teck » (Al-Hamadānīi 1973 : 30).

À Bașra, ville fondée en $635^{21}$, on créa une première mosquée, simple enclos de briques cuites avec des portiques en bois de teck (Terrasse 1971: 166). Car, dit Abū Bakr alHudalalī, dans la rivalité entre les villes de Bașra et de Kūfa ${ }^{22}$, «nous avons plus que vous $\mathrm{du}$ teck, de l'ivoire, du brocart, des cours d'eau bruyants et des revenus fonciers ». (Ibn al-Faqīh al-Hamadānī, début du xe s., $1973: 146$, repris p. 230) ${ }^{23}$. En effet, le teck était un bois assez commun, bien que considéré comme précieux, dans cette ville de Bașra qui, pendant plusieurs siècles, fut le principal port recevant en quantité les grumes de teck d'Inde, comme le dit Mas'ūdī au Xe siècle (1961, II : 324). Lorsque Abū Mūsā († en 662 ou 672) en devint gouverneur, il bâtit la mosquée de brique crue, de même que l'hôtel de l'émirat. 'Ubayd Allāh b. Ziyād, gouverneur de Bașra, Kūfa et du Hुorāsān sous les premiers califes, bâtit la mosquée en brique cuite et plâtre, avec une "toiture ${ }^{24} \mathrm{de}$ teck (al-Hamad̄ānī 1973 : 228).

Ibn Jobāir, le valencien voyageur et géographe du $x^{e}$ siècle, décrit « la vieille mosquée

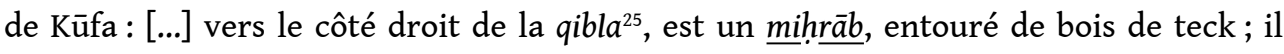
s'élève au-dessus de la travée pareil à un petit oratoire, c'est le mihrāb du Prince des Croyants, 'Ali, fils d'Abū Ṭâalib» (1951:241)», c'est un lieu sacré pour les Chiites. Plus loin il décrit « un autre petit miḩrāb encadré de bois de teck, qui est l'endroit d'où jaillit le tannūr qui fut un signe pour Noé » (idem). Ces deux mihrāab sont des lieux sacrés. Deux siècles plus tard, vers 1330, ibn Bațțūta, confirme et cite cette description d'Ibn Jobāir (1982, I : 430).

34 En effet, Ibn Bațtūta décrit la grande mosquée de Kūfa, qui est une des métropoles de l'Iraq : «Elle possède, écrit-il, de nobles restes et parmi ceux-ci une cellule en face du

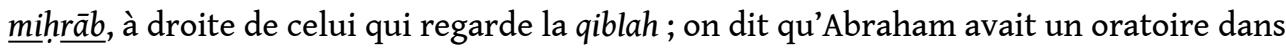
cet endroit; tout à côté, on voit un autel, entouré de planches de bois de teck, il est

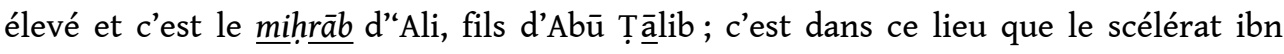
Moljam frappa 'Ali, et le public s'empresse d'y venir prier. » (1968, II : 94). Il ajoute et précise « dans l'angle, au bout de cette nef, il existe une petite chapelle, entourée aussi de bois de teck, et l'on dit que c'est la place où la fournaise déborda (ou bien, l'orifice bouillonna) lors du déluge de Noé que cite le Coran (XI, 42)» (idem, II : 95). 
La grande mosquée de Damas ${ }^{26}$, l'une des merveilles de l'art musulman au viII siècle « fut construite en 706, par le calife omeyade al-Walìd (règne 705-715), en marbre et mosaïque et couverte de bois de teck, ornée de lapis-lazulis ${ }^{27}$ et d'or... » (Burns 2005, Le Strange 1965 : 236). Elle subit ensuite plusieurs tremblements de terre et des incendies après lesquels elle fut totalement reconstruite. Deux siècles plus tard, al-Hamadāāi l'admire (1973 : 133) et al-Muqaddasĩ (c. 945-991, le géographe syrien qui écrit en 985, en donne une description assez précise 84 ans avant un premier incendie en 1069 (1963 : 169 n. 98). Elle a malheureusement brûlé en 1893 dans un violent incendie qui engloutit les plafonds de la salle de prière.

De nos jours, le plus ancien minbar (chaire), encore intact, est celui de la Grande Mosquée de Kairouan, daté du IX ${ }^{\mathrm{e}}$ siècle (vers 863). C'est l'émir Ibrāhīm ibn al-Ag̀lāb qui fit venir du teck, sāj, de Bag̉dād pour construire ce minbar (Lombard 1971 : 176, n. 34). «Il s'agit d'une chaire, entièrement réalisée en bois de teck importé d'Orient, en forme d'escalier à onze marches, par lesquelles on accède au siège supérieur. [...] Ce minbar est considéré comme un joyau de l'art classique musulman. Les pièces de ce bâti, les panneaux, les contremarches, le coffre et le dossier du siège sont entièrement décorés, soit d'entrelacs géométriques, soit de formes végétales » (Marçais $1962: 50$ ).

$\mathrm{Au} \mathrm{x} \mathrm{x}^{\mathrm{e}}$ siècle, «le teck apparaît dans les incrustations décorant le minbar de la grande mosquée de Cordoue» (Lombard 1972: 149, n. 172). Elle fut construite au viII siècle sous le règne de l'émir 'Abd ar-Rahmmān I. Un autre beau minbar se trouve dans la mosquée seldjoukide dite d"Ala-Eddīn à Konya ${ }^{28}$, en Anatolie, il date de 1155. Il est fait de deux bois précieux, l'ébène, Diospyros sp., bois africain et le teck, bois indien (Huart $1898: 10)$.

38 A propos de la longue durabilité du teck, sir George Watt donne l'exemple d'un arbre qui offre son ombre depuis au moins 2000 ans, dit-il, à l'une des nombreuses grottes de Karli, site extraordinaire dans le Maharashtra, au sud de l'Inde (Watt $1966: 1078$ ).

\section{Le teck dans les constructions citadines et artisanales}

Le teck était, certes, d'abord un bois de marine, mais il entrait aussi dans la menuiserie des constructions prestigieuses, mosquées, palais et petits meubles précieux, etc.

Ce fut le cas de la création de Sāmarrā par le $8^{\mathrm{e}}$ calife abbasside Abū Išāq, $3^{\mathrm{e}}$ fils d"Abbās ben Hārūn ar-Rašīd, et surnommé al-Mu'tașim bi-llāh, (né en 796 et mort le 5 janvier 842 à Sāmarrā). Il fonda cette ville et l'inaugura au début du IX $\mathrm{x}^{\mathrm{e}}$ siècle. Il y fit construire la première grande mosquée dite $d u$ Vendredi, près de la rive gauche du Tigre (Le Strange 1900 : 54). Pour ce faire «il donna par écrit l'ordre de faire venir des artisans [...], de faire transporter toutes sortes de bois, du teck notamment, des troncs de palmiers, de Bașra ${ }^{29}$ et de ses environs (al-Ya'aqūbī, † c.905, 1937: 49). On utilisa également le teck dans les boiseries du palais du Djawsāq dans la Sāmarrāa abbasside au IX ${ }^{\mathrm{e}}$ siècle.

41 Mais, on utilise également ce bois pour les portes des belles maisons. À Médine, 'Uțmān, le $3^{\text {e }}$ calife (577-656), construit un hôtel en pierre et chaux, dont les portes étaient en bois de teck et de thuya (Ma-s'ū ùi 1961 III : 616, 1578). Au Xe siècle, à Damas, le teck des portes des maisons princières vient de Bașra ou d'Aden par mer (al-Ḥamad̄ānī 1973 : 133). 
Ce sont donc des constructions citadines qui utilisent le teck; c'est ce que l'on voit à Sirnāf comme le dit ibn Ḥauqal ( $\left.x^{e} s.\right)$, dans sa description des villes du Golfe Persique. Il note que «cette ville est le grand port du golfe; c'est une cité importante dont les maisons sont construites en bois de teck et se touchent sans interruption jusqu'à une montagne qui surplombe la mer...» (1964 1 : 47) ; plus loin, il la compare à Širāz et dit qu'elle est aussi vaste que cette ville et il reprend sa description de façon plus complète. Ses maisons sont construites en bois de teck et d'une autre espèce de bois qu'on importe du pays des Zanj (Afrique orientale). Les maisons ont plusieurs étages comme à Fosțāṭ (Égypte) ; la ville est située au bord de la mer ; les maisons sont enchevêtrées ; le nombre des habitants est considérable ...»(1964 II : 277). En effet, cette ville, principalement habitée par des Persans et des Arabes commerçants, est devenue le port d'arrivée et de départ du commerce avec l'Inde et plus loin la Chine. Elle prît de l'importance car Bașra, dans le fond du Golfe, s'enlisait peu à peu du fait des alluvions que le Tigre charriait. Les marchandises, dont le teck restait une des plus importantes, débarquaient donc à Sīrāf et étaient transportées sur des bateaux de moindre tirant d'eau à Bașra. Sīrāf était avant tout le port des Persans (Sauvaget 1948 : XXXIX).

Près d'Abādān, ville du Hुuzistan en Iran, sur une île du Šoțt el-ārab qui est le chenal du delta commun du Tigre et de l'Euphrate débouchant dans le golfe Persique, Nasir iHosrau, persan qui voyagea de 1035 à 1052, ayant pris la mer dans cet endroit, voit dans le lointain ce qu'il nomme un Hošāb, sorte de phare et de poste d'observation, sur l'eau, qui signale aux bateaux des dangers proches naturels ou de pirates. "Ce Hošăb est fait de quatre piliers de teck ${ }^{30}$ mis en carré, plus large au niveau de l'eau et plus étroit audessus et formant une plate-forme sur laquelle on a établi une sorte de loge carrée et fermée par des verres qui protègent $d u$ vent les feux allumés et où se tient un guetteur... » (Sheffer $1881: 246$, dans sa note, cite de nombreux auteurs qui ont décrits ces Hošăb ou phares implantés sur les côtes).

44 Près de Mossoul (Mūṣul), ibn Ḥauqal qui visite et décrit très précisément les lieux intéressants du Moyen Orient à cette époque, $\mathrm{x}^{\mathrm{e}}$ siècle, parle avec admiration des moulins à eau, urūb, au milieu du Tigre (Dijlah). Cette sorte de moulins, dit-il, n'avait pas leur pareil en aucun point de la terre ; ils étaient installés au milieu du fleuve au cours rapide, attachés par des chaînes en fer. Chaque moulin avait quatre meules et, dans l'espace d'un jour et d'une nuit, deux meules pouvaient moudre cinquante charges de mulets. Ces moulins étaient en bois et en fer et parfois on utilisait du bois de teck. On trouvait à Balad, à sept parasanges de Mossoul, une grande quantité de ces moulins qui tournaient continuellement ; la farine produite était expédiée en Iraq. L'action funeste d'ibn Hamdan ${ }^{31}$ « n'en a laissé subsister aucun » (cf. Canard $\left.1951: 117\right)$. Voilà pourquoi, ibn Ḥauqal parle au passé (1964, I : 213).

45 En Inde également, selon 'Abul-Faradj (qui écrit en 988), le temple de Mānkīr, dans le sud-ouest de la province de Haïderabad, «capitale où réside le Ballahrā, est construit en bois de teck, roseau et en différentes espèces de bois ». C'est un temple très riche où sont " vingt mille Bouddhas en toutes espèces de matières précieuses » (in Ferrand 1907, I : 119). 


\section{Les usages artisanaux du teck à Goa} religieux, atteint de judāmm ${ }^{32}$ en Iraq, maladie qu'il est bien difficile de déterminer C'était à l'époque du calife Harūn al-Rašīd (c.766-809) «J'ai entendu crier dans la maison d"Abd Allāh ; on m'a dit qu'il était atteint de judāamm, que son corps se tuméfiait et devenait tout noir, j'ai été voir Rašīd pour le lui dire, mais je n'avais pas fini de parler qu'ils vinrent me dire qu"Abd Allāh était mort : tout de suite, je leur ai dit de hâter l'enterrement. J'ai moi-même dit l'oraison funèbre, ... puis comme ils descendaient le cercueil dans la fosse, un éboulement se produisit et le cercueil roula avec la terre et une odeur intolérable se répandit; à ce moment-là, des gens passaient portant des fascines, je fis apporter toute la charge et la fit placer dans la fosse, mais elle ne fut pas mieux étayée et un second éboulement eut lieu. Je demandai alors des planches de teck pour couvrir la fosse... et l'on jeta de la terre par-dessus » (1991, IV : 1019-1020, §§ 2504-2505).

52 
le teck, sāj, en collyre, après une certaine préparation, on peut aussi «en calmer l'inflammation des tempes» (le mal de tête). Il note ensuite l'usage de "son fruit dont on tire une huile qui sert à sophistiquer les vessies de musc ; on les farcit de manière à ce que le mélange n'apparaisse pas et que le poids s'en trouve augmenté $»^{33}$ (1987, II : 233).

\section{D'où venait le teck chez ces Arabes}

53 Ce bois venait, au plus près, des Ghatts ou collines occidentales de l'Inde, notamment le Konkan qui est la région côtière qui va de Bombay à Goa ; pour les Arabes géographes c'est le pays du teck (Ferrand 1907, I : 29, 1196; Sauvaget 1948: 51); mais aussi le Malayalam ou Malabar, plus au sud, où on le trouve près d'une ville appelée Kūlam, sur la côte sud occidentale de l'Inde (Yakut, 1179-1229, in Ferrand 1907, I : 225). Ainsi, des côtes occidentales nord et sud de l'Inde, le teck gagnait par bateaux la Mésopotamie, aujourd'hui l'Iraq, par le Golfe Persique, et l'Égypte par la Mer Rouge puis par le canal de l'Émir des Croyants ${ }^{34}$.

Ce bois mi-lourd était onéreux, essentiellement réservé aux constructions navales soignées et constructions terrestres prestigieuses comme le rapporte Abū Yūsuf (VIII ${ }^{\mathrm{e}}$ s.), dans son Livre de l'impôt ${ }^{35}$; parlant des peines appliquées au voleur de planches de teck, il écrit qu'il n'y a pas d'amputation pour ce vol ou celui d'autres bois d'œuvres à moins qu'ils ne soient transformés en vases ou en portes (1921:266). C'est-à-dire qu'à cette époque, à Kūfa, on le réservait presqu'essentiellement aux constructions terrestres prestigieuses.

Le teck arrivait ainsi dans toute sa longueur à l'entrepôt de bois, sāḥil al-ḥašabāt (Lombard 1971 : 175). Il arrivait dans la ville de Bașra, en Mésopotamie, et en quantité selon le bağdādī Mas'ūdī au Xe siècle (c. 896-956), car c'est un port et une ville riche (1961, VI : 276) ; plus loin, Mas'ūdī signale des planches de teck pour couvrir une fosse tombale ; cela a probablement à voir avec le mort de judām (id., vol. IV : 1019-1020, 2505 $=\mathrm{VI}: 299)$ dont on a parlé plus haut.

Pour Mas'ūdī, l'Inde est le pays où pousse le teck avec de nombreuses plantes à parfum, comme nous l'avons vu. (1961, I : 97). Ce bon connaisseur des embarcations note qu'il y a des différences de construction des vaisseaux en Méditerranée et celle des navires de l'océan indien, qu'il nomme mer d'Abyssinie, sans plus s'attarder. Ce que nous faisons, par contre. En effet la marine arabe de Méditerranée, héritière de la marine romaine, clouait les bordés sur les varangues, alors que dans l'Océan Indien, soumis aux moussons et devant aborder fréquemment, on cousait et recousait les bordés les uns aux autres avec les cordages solides et imputrescibles des palmiers. Ainsi, en hiver, comme l'a vu mon père vers 1930, on décousait les bordés et on les recousait à la belle saison. Ibn Bațtututa décrit précisément la fabrication, par les femmes, aux Maldives, des cordages avec ce qui enveloppe la noix de coco (kanbar, c'est le coir) et, pour leur solidité, « on exporte, ces cordages en Chine, en Inde et au Yémen » (1822, III, 228).

À la même époque, $\mathrm{x}^{\mathrm{e}}$ siècle, un petit ouvrage, intitulé 'adjāib al-Hind (Les merveilles de l'Inde) présente, sous forme de conte, les péripéties, si je puis dire, d'une longue planche de teck, dont on ne dit pas l'usage, mais qui devait être de grande valeur (Devic 1878 : 123). 

situer, sur les lieux où l'on trouve les arbres de teck. Al-Mas'ūdì qui écrivit le très célèbre et gros ouvrage Les prairies d'or, Murūğ ad-dahab, au xe siècle, connaît bien les bateaux et leur construction et peut détailler les différences de construction des vaisseaux en Méditerranée et en mer d'Abyssinie (1961, I : 365) comme nous l'avons vu. Il cite aussi à plusieurs reprises les lieux où l'on trouve le teck, sāj, arbre, (id., II, 1965 : 324, 338, 853, 899 ; III, $1971: 12,56$ ), et son bois (VI : 276, 299). Orient, G. Ferrand note, au Voyage de Šams ed-Dīn ad-Dimašqi (1256-1327) vers 1325, d'autres lieux où pousse le teck; ce grammairien de Damas voit des arbres dans une île qu'il nomme Malay ${ }^{36}$, au large du Cambodge : « Sur cette île croît l'arbre de teck (sāj) qui devient grand et fort [...] on en construit des vaisseaux formés d'une seule planche ${ }^{37}[. .$. la longueur des planches est de 40 coudées, sur une largeur de 7 coudées " (in Ferrand 1914, II : 384). Ce qui donne, la coudée étant évaluée à environ $0,50 \mathrm{~m}$, des planches d'environ $20 \mathrm{~m}$ de long ce qui est tout à fait possible, sur 3,50 m de large, ce qui est beaucoup moins possible, à moins d'assemblage. Plus loin, Dimašqī voit que Poulo Condore, île au large du sud-Viêtnam, produit principalement des arbres de teck et des manguiers (id. p. 387).

\section{Les noms du teck, Tectona grandis L.f.}

60 Les noms du teck sont variés. En Inde, et en hindou du Bengale occidental, l'espèce est connue sous le nom de segun, seg ou sag, et le Mahrati sāg puis sāj arabe (Yule \& Burnell 1903), du sanscrit saka ${ }^{38}$. Yule pense même que sagalina peut être le nom grec du teck dans le Périple de la mer Erythrée, le plus ancien texte de ces régions. Le français teck et l'anglais teak viennent du tamoul tekku via le portugais teca. De la même racine sont le Malayalam tekka (Yule \& Burnell 1903) et le Kannada tega. Dans tout l'archipel insulindien, un seul nom, djâti, jatii désigne le teck.

Les noms de l'arbre relèvent ainsi de trois racines, au moins : tekku ou tek, tati ou techati (ce dernier nom étant d'ailleurs celui employé dans la région de Kalinga ${ }^{39}$ ), et segun, seg, sag.

62 Les naturalistes ont très tôt porté attention au teck. Ainsi Bontius ${ }^{40}(1592-1631)$ médecin, lui avait donné le nom de Quercus indica, "chêne indien ", lui trouvant des qualités similaires au chêne, mais il n'en avait pas vu les feuilles, grandes, arrondies et sans aucune comparaison possible avec celles de chênes. D'autres auteurs le désignent sous le nom de « chêne de Malabar » ou des Indes. Hendrik van Rheede (1636-1691) le décrit sous le nom de Theka (de thekku, nom du teck en Malayalam) dans son Hortus indicus Malabaricus qui parait vers la fin du XvII e siècle (Figure 2). Il note (pp. 57-58) au chapitre de l'arbre, qu'il pousse en abondance au Malabar, que son bois est semblable à celui du chêne, et qu'on l'emploie pour la construction des navires. Il indique aussi que les jeunes feuilles rougissent et teignent les tissus de soie et de coton, que les indigènes mangent ces feuilles, qu'ils en font, en les cuisant avec du sucre, un sirop qui soignent les aphtes. Rumphius (1627-1702), allemand au service de la Compagnie hollandaise des Indes orientales et botaniste, latinise le terme djati d'Amboine en jatus, ou kiate en néerlandais dans son ouvrage Het Amboinsche kruidboek, (vol. 3, pp. 34-36, planche XVIII). Linné enfin le baptise Tectona grandis (1758), son nom scientifique actuel. 
63 englobe l'Inde, la Birmanie, le Siam. À Java et certaines des petites îles de la Sonde, il aurait été introduit vers l'an 200 par des Indiens adeptes de Vishnu pour lesquels c'était un arbre sacré. Aujourd'hui l'arbre djati, son nom local, se déploie en véritables plantations dans cette île.

ès le $\mathrm{XVIII}^{\mathrm{e}}$ siècle, les Portugais l'introduisent en Afrique et en Amérique tropicale, et au XIX ${ }^{e}$ siècle les Français le plantent en Afrique centrale et en Guyane. Il devient aujourd'hui une espèce de reboisement importante et même prioritaire tant en Asie qu'en Afrique et en Amérique tropicales, pour les qualités de son bois et sa croissance rapide, bien meilleures que celles de l'eucalyptus.

Après de nombreux usages prestigieux utilisant les propriétés très particulières de ce bois pendant près de dix siècles, nous avons vu que ce bois était également connu, aux $\mathrm{XII}^{\mathrm{e}}$ et XIII ${ }^{\mathrm{e}}$ siècles, jusque dans les terres les plus occidentales des Musulmans, l'Espagne, et l'on voit qu'aujourd'hui ce bois sert à faire des chaises, des tables et autres meubles de jardins, fort utiles certes, mais pour lesquels on pourrait utiliser des bois plus locaux.

\section{BIBLIOGRAPHIE}

Ābū Yūsuf, Ya'qūb ibn Ibrāhīm al-Anșārī 1921 - Kitāb al-Kharāj, Le livre de l'impôt foncier. Trad. et notes par Edmond Fagnan. Paris, Geuthner, xvi-352 p.

Al-Ḥamad̄ānī, Ibn al-Faqīh 1973 - Abrégé du livre des Pays. Trad. Henri Massé. Damas, Institut français.

Al-Mas' ūdī (1928-30) 1961-1995 - Murūğ ad-dahab. Les Prairies d'or. Barbier de Meynard et Pavet de Courteille, éds et trads., revues par Charles Pellat, Paris, Geuthner, 6 vol.

Al-Muqaddasī 1963 - Ahsan al-taqāsīm fì ma rifat al-aqālīm. La meilleure répartition pour la connaissance des provinces. Trad. partielle annotée par André Miquel. Damas, Institut Français.

Aloudat N, Carayon A. \& Giovannoni V. (Ed.) 2016 - Aventuriers de la mer, VIIe-XVIIe siècles : de Sindbad à Marco Polo : Méditerranée, océan Indien : [Exposition, Paris-Institut du Monde Arabe, 15 novembre 2016-26 février 2017 ; Marseille-MuCEM, 6 juin-9 octobre 2017]. Marseille, éditions du MUCEM ; Paris, Institut du monde arabe, $223 \mathrm{p}$.

Al-Ya'aqūbī 1937 - Les pays. Le Caire, IFAO.

Consigny 1935 - Où planter du teck en Indochine. Bulletin Economique de l'Iindochine 38, décembre : 1009-1022.

Aubaile-Sallenave F. 1969 - Les bateaux Moliceiros de la lagune d'Aveiro (Portugal). Mémoire de maîtrise. Paris, Sorbonne, $110 \mathrm{p}$.

Aubaile-Sallenave F. 1974 - Les matériaux de construction des embarcations du Viêtnam. Doctorat de 3e cycle, Paris, EPHE, 6e section.

Aubaile-Sallenave F. 1982 - Les voyages du Henné. JATBA XXIX : 123-178. ; 
Aubaile-Sallenave F. 1984 - L'agriculture musulmane aux premiers temps de la conquête : apports et emprunts. JATBA XXXI : 245-256.

Aubaile-Sallenave F. 1987 - Bois et bateaux d'Indochine. Louvain, SELAF.

Aubaile-Sallenave F. 1990 - Le sel de l'alliance. JATBA XXXV : 303-323.

Aubaile-Sallenave F. 1994 - La mousson, pluie des agriculteurs, vent des marins, Revue d'ethnoécologie 5. doi.org/10.4000/ethnoecologie.1834

Aubaile-Sallenave F. 2005 - Le thé, un essai d'histoire de sa diffusion dans le monde musulman. In : Marín M. \& de la Puente C. (Ed.) El banquete de las palabras : la alimentación en los textos àrabes. Madrid, CSIC : 153-200.

Bontius 1658 - Historiae naturalis et medicae Indiae orientalis, publiée par le médecin Guillaume Pison de Leyden. Amsterdam, Ludovicum \& Danielum Elzevirios, $6 \mathrm{v}$.

Burns R. 2005 - Damascus. A History. New York, Routledge, 386 p.

Canard M. 1951-1967 - Histoire de la dynastie des Hamdani-des de Jazira et de Syrie, t. I. Faculté des Lettres d'Alger, xvi et $863 \mathrm{p}$.

Carreira E. 2016 - L'empire portugais d'Asie et la Méditerranée aux XVIe et XVIIe siècles. Genèse d'une mondialisation des échanges. In : Aventuriers des mers, VIIe-XVIIe siècles : 157-166. Marseille, MUCEM.

Casson L. 1989 - The Periplus Maris Erythraei. Text with Introduction, Translation and Commentary. A Princeton University Press E. Book.

Dauphinot G.1905 - Les forêts de teck au Siam. Bulletin Economique de l'Indochine : 625-636.

Devic M. 1878 - Les Merveilles de l'Inde. Paris, Alphonse Lemerre.

Dozy R.P. A. (1881) 1967 - Supplémemt aux dictionnaires arabes. Leyden, Paris, Brill, Maisonneuve et Larose. 2 vol.

Ferrand G. 1907 - Les géographes arabes et Madagascar : les îles Ramny, Lâmery, Wâqwâq, Qomor des géographes arabes et Madagascar. Journal asiatique 10 : 433-566.

Ferrand G. 1913-14 - Textes arabes relatifs à l'Extrême Orient. Paris, E. Leroux, 2 vol.

Flecker M. 2000 - A ninth century AD Arab or Indian ship-wreck in Indonesia. first evidence for direct trade with China. International Journal of Nautical Archaeology 9, 38 p.

Flecker M. 2008 - A ninth century AD Arab or Indian shipwreck in Indonesia Waters: Addendum. International Journal of Nautical Archaeology 37, 3 p.

Hawkins C.W. 1981 - Les Boutres, derniers voiliers de l'Océan Indien. Lausanne, Edita.

Huart C. 1898 - La Ville des derviches tourneurs [Konia] [précédé d'une notice par Charles Simond]. Paris, Plon, $32 \mathrm{p}$.

Ibn al-'Awwām 1864-1867 - Kitāb al-Filāḥa, Le livre de l'agriculture. Traduction Clément-Mullet. Paris, Albert L. Herold, 2 .

Ibn al-Beițār 1877-1883 - Kitāb ǧāmit al-mufradāt « Traité des Simples », Lucien Leclerc, trad. Paris, Institut du monde arabe, $3 \mathrm{v}$.

Ibn Bațțūta (1822-1883) 1968 - Tuhfat al-nuzzār fi gharib al-amsār wa-ajāib al-asfār. Tuhfat al-nuzzār "Voyages". B.R. Sanguinetti \& Charles Defrémery (éds et trad.) Larousse 7 vol. Paris, Anthropos. 
Ibn Bațțūta 1982 - Voyages, 1. De l'Afrique du nord à la Mecque, 2. De la Mecque aux steppes russes, 3. Inde, Extrême Orient et Soudan. Paris, Maspéro. (La Découverte).

Ibn Ḥauqal 1964 - Le Livre de la configuration de la terre, trad. J.H. Kramers et G. Wiet. Paris, Maisonneuve et Larose.

Ibn Jobaïr (Ğubayr) 1951-1956 - Voyages. M. Gaudefroy-Demombynes, trad. et notes. Paris, Geuthner. 3 vol., le $4^{\mathrm{e}}$ vol., 1965, comprend les addenda, les index, des notes additionnelles et les tables, élaboré par M.P.L. Martin Morice, et revu par Henri Massé et Gaston Wiet.

Kazimirski A. de Biberstein (1860) 1980 - Dictionnaire arabe-français. Beyrout, Librairie du Liban, $2 \mathrm{v}$.

Kerlouégan J. \& Bing Zhao 2016 - Les expéditions de Zheng He (1371-1433) ou le rêve chinois d'un empire maritime. In : Aventuriers des mers, VIIe-XVIIe siècles : 119-126. Marseille, Mucem.

Le Strange G. (1900) 1966 - The lands of the eastern caliphate. London, Cass.

Levi-Provençal É. (1953) 1969 - La civilización àrabe en España. Madrid, Espasa-Calpe.

Lewicki T. 1936 - Les premiers commerçants arabes en Chine. Rocznik Orientalistyczny 11 : 173-186. Lombard M. (1971) 1980 - L'islam dans sa première grandeur. Paris, Flammarion. (Champs).

Lombard M. 1972 - Espaces et réseaux du haut moyen âge. Paris, Mouton.

Marçais G. 1962 - L'art musulman. Paris, PUF.

Miquel A. 1980 - La géographie humaine du monde musulman jusqu'au milieu du $11^{e}$ siècle, II. Paris, La Haye, Mouton.

Pelliot P. 1959 - Notes on Marco Polo. Paris, Imprimerie nationale, Adrien-Maisonneuve, 3 v.

Picard C. 2016 - Al-Andalus, une grande puissance maritime médiévale ? In : Aventuriers des mers, VIIe-XVIIe siècles :135-142. Marseille, Mucem.

Piétri J.B. 1943 - Voiliers d'Indochine. Saigon, Société des imprimeries et librairies indochinoises, $130 \mathrm{p}$.

Planhol X. de 2000 - L'Islam et la mer. La mosquée et le matelot. VIIe-XXe siècle. Paris, Perrin. 658 p.

Robequain C. 1946 - Le monde malais. Paris, Payot.

Rumphius G.E. 1741-1755 - Het Amboinsche kruidboek, publié par le médecin hollandais Jean Burman, qui a aussi traduit le texte allemand en latin. Amsterdam. 5 parties en 6 vol., le 7e étant un index.

Sallenave P. 1949 - Le teck. Neptunia, 15 : 34-37.

Sauvaget J. 1948 - Relation de la Chine et de l'Inde, rédigée en 851 par le marchand Suleïman. Paris, Les Belles lettres, $41+79$ p..

Schefer C. 1881 - Sefer Nameh, Relation du voyage de Nāsir-i Khosrau en Syrie, en Palestine, en Égypte, en Arabie et en Perse pendant les années 427-444 (1035-1052). éd. et trad. Paris, Leroux. Terrasse H. 1971 - Les débuts de l'architecture musulmane (622-750) [compte rendu] : Le journal des savants $3: 161-186$.

Van Rheede tot Drakestein H. 1637-1691 - Hortus Indicus Malabaricus. Amsterdam, Someren \& Dyck.

Watt G. (1908) 1966 - The commercial products of India, being an abridgment of "the dictionary of the economic products of India. New Delhi, Today \& tomorrow printers \& publishers. 
Ya‘aqûbī 1937 - Kitāb al-buldān Livre des pays. G. Wiet, trad. Le Caire, IFAO, 291 p.

Yule H. 1903 - The Book of Ser Marco Polo. London, John Murray, 2 v.

Yule H. \& Burnell A.C. (1903) 1984- Hobson-Jobson. A Glossary of col-loquial anglo-indian words and phrases, and of kindred terms, etymological, historical, geographical and discursive. $4 \mathrm{è}$ éd. rev. par W. Crooke. New Delhi, Munshiram Manoharlal, $1021 \mathrm{p}$.

\section{NOTES}

1. FRA : Formation à la recherche en anthropologie.

2. CEDRASEMI : Centre de recherche sur l'Asie du sud-est et le monde insulindien.

3. En particulier Aubaile-Sallenave 1982, 1984, 1990.

4. A dictionary of Iraqi Arabic, English-Arabic, Washington, Georgetown University Press, 1944, au mot teak, il donne sāj, et en exemple la phrase suivante : our dining-room table is teak (notre table de salle à manger est en teck).

5. On évalue la taille maximum des bateaux chinois venant dans le Golfe persique à $60 \mathrm{~m}$ de long pour les plus gros bateaux de la flotte (Kerlouégan et Bing Zhao 2016 : 121).

6. D'autres produits étaient commercialisés avec le teck, tels que le cuivre, les bois d'ébène et de Dalbergia sissoo... (Casson, pp. 72 et 73).

7. Tous le disent, comme par exemple Ibn Khordadbeh (in Ferrand $1913: 29$ ).

8. Ho Chi Minh ville, l'ancienne Saïgon, capitale du Sud-Vietnam, est en grande partie construite sur des pilotis de teck, enfouis dans l'eau et toujours en état (P. Sallenave, info. orale)

9. Ville de Mésopotamie (aujourd'hui Iraq), sur le Tigre, bâtie près de la ville grecque de Séleucie. Construite par les Parthes arsacides, elle devint leur capitale, puis fut celle des rois sassanides.

10. Voir mon texte de la mousson, 1994, dans lequel j'ai inséré 8 photos de boutres arabes traditionnels.

11. Procédé consistant à couper une lanière d'écorce sur toute la périphérie du tronc, empêchant ainsi la montée de la sève.

12. On sait que l'Irak possédait de grandes plantations de cocotiers.

13. Lors des attaques vikings en 844 , au sud de l'Espagne les arsenaux d'al-Andalûs se déplacèrent sur les rives du Guadalquivir, très en amont de Séville, dans les environs de Cordoue où les navires furent construits. Plus tard, des marins berbères, venant de la rive sud de la Méditerranée, apportèrent leur savoir-faire tant en matière de charpenterie que pour la navigation (Picard $2016: 137$ ).

14. Ce canal de liaison était très important pour le commerce entre l'Égypte, puis la Méditerranée et l'Orient et l'Extrême Orient. Il va du nord-occidental de la mer Rouge, de Qolzum à Fosțāt Le Caire, port de l'une des branches du Delta du Nil, qui se jette dans la Méditerranée non loin de Damiette ( $c f$ Lombard 1971, carte p. 24). Ce canal existe depuis longtemps puisque Ptolémée II vers 250 av. J.-C. le restaure. Au cours des mille années qui suivirent, il fut successivement modifié, détruit et reconstruit, notamment par Amr ibn al-As qui conquiert l'Égypte en 639-642 (en 640, il bat les troupes byzantines à Héliopolis et, en 642, il prend Alexandrie). Ce canal devint alors le «canal du Commandeur des croyants" en hommage au calife 'Omar ibn al-Hुațāa (584-644) qui administre l'Égypte avec "sagesse" (sic). Sous son règne, de nombreuses antiquités et reliques d'Égypte considérées comme païennes furent détruites. Il envisagea notamment de détruire les Pyramides. Ce canal fut finalement détruit au VIII ${ }^{\mathrm{e}}$ siècle par le calife al-Manșūr (714-775) pour isoler la ville de Médine et éviter ainsi le risque d'une attaque.

15. Voir Aubaile-Sallenave 2014.

16. L'ensemble des marchandises de ce bateau a été acheté par le Musée de Singapour (Flecker. 2008) 
17. Dans Aubaile-Sallenave 2014 figurent des photos de bateaux arabes et de boutres, qui sont équivalents au dhow.

18. Niche insérée dans le mur de la qibla donnant la direction de la Ka'aba vers laquelle on doit se tourner pour prier.

19. Ce sont les premiers califes :'Umar ibn al-Hुațāāb, second calife qui vécut de 581 à 644 , 'Uțmān ibn 'Affān lui succède de 644 à 656 quand il a été assassiné.

20. C'est, dans la mosquée, une petite pièce où le souverain se retire pour prier.

21. Deux siècles plus tard, cette ville était encore importante, puisque le Chau Yu Kua, ouvrage chinois, la cite en 1225 (in Pelliot 1959, notes, vol.1, p. 89).

22. Cette rivalité entre les deux villes est une joute verbale et écrite assez curieuse que plusieurs auteurs ont repris, ici al-Hamadān̄ī, et aussi son presque contemporain al-Mas'ūīi (1961, IV, paragraphes 2481-2484).

23. A la fin de son texte sur l'Irak, Ibn al-Faqīh al-Hamadānī (début du IX ${ }^{\mathrm{e}} \mathrm{s}$.) reprend l'éloge de Bașra qu'il met dans la bouche de H̦ālid b. Șafwān, un député de Bașra, « Deux chasseurs d'entre nous partent de grand matin : l'un apporte l'alose (šabbūṭ) et le šim (probablement le barbeau, très présent dans le Tigre, selon Miquel, 1980, II, 367, n.6)), l'autre apporte les oiseaux et l'autruche mâle; nous sommes les gens qui possédons le plus d'ivoire, de bois de teck, de Hazz (étoffe), de brocart, de montures au pas doux et rapide, de vierges aux regards obliques; nos maisons sont d'or et notre fleuve - le Tigre - est une merveille ». (Al-Hamad̄ānī 1973 : 146 et 233).

24. On ignore si le terme « toiture » désigne la charpente ou la couverture ou les deux.

25. Quand les Musulmans prient, ils se tournent vers un mur, la qibla, dans laquelle est une niche, $\underline{m i h} \underline{r a ̄ b}$, qui donne la direction de la Ka'bah, à la Mecque.

26. Le site de cette mosquée était auparavant celui de la cathédrale St Jean et les Musulmans conservèrent le petit mémorial dédié à saint Jean le Baptiste (voir Ross Burns 2005).

27. Le lapis-lazulis, pierre d'un bleu intense, venait d'Asie centrale et plus précisément d'Afghanistan. Il était déjà connu dans l'Egypte de Ramsès II (1301-1236 avant J. C.).

28. Konya est la ville où sera fondée, au XIII ${ }^{\mathrm{e}}$ siècle, la confrérie des Mawlāwī, derviches tourneurs, disciples de Djalāl al-Dīn Rūmī, le poète et mystique persan. Né à Balkh en 1207 (actuel Afghanistan), il s'exile, fuyant l'invasion des Mongols (1216-1218), en Turquie où il meurt à Konya en 1273.

29. On sait par Mas'ūdī ( $\mathrm{X}^{\mathrm{e}} \mathrm{s}$.) que Bașra était le port d'arrivée des troncs de tecks des forêts de la côte occidentale de l'Inde et qu'il en arrivait de grande quantité. (II § 853)

30. Le teck, ici, se justifie parfaitement, car il se conserve fort bien dans l'eau.

31. La dynastie hamdanide (حمدانيون; Ḥamdāniyyūn) est une dynastie arabe d'émirs chiites (890-1004). Les capitales de cet émirat furent Mossoul et Alep. Cette dynastie apparait dans un contexte d'affaiblissement du pouvoir central abbasside, qui voit dans cette période $\mathrm{du} \mathrm{X}^{\mathrm{e}}$ siècle l'émancipation et l'affirmation de petites dynasties qui s'emparent des pouvoirs temporels et spirituels du califat à une échelle locale ou régionale.

32. Judām. Kazimirski traduit le mot par éléphantiasis, Pavet de Courteille, Barbier de Meynard (dans leur édition de Mas'ūīi) et Pellat qui révise le texte, le traduisent par lèpre. Mais ces deux maladies ne noircissent pas le corps qui est dit se tuméfier et devenir tout noir ; ne serait-ce pas plutôt la peste, d'où l'enterrement très rapide, mais là non plus, le corps ne noir-cit pas. Dozy, dans son Dictionnaire arabe reprend les deux maladies citées et y ajoute le feu de St Antoine, qu'on appelait aussi Mal des Ardents qui était l'ergotisme, c'est à dire un champignon de la famille des Ascomycètes, du genre Claviceps purpurea, l'ergot du seigle, de l'orge, du froment et d'autres céréales et qui provoque des hallucinations, des douleurs terribles aux extrémités; puis le corps se dessèche et devient tout noir. Mon collègue, le médecin Alain Froment, m'écrit qu'il est très difficile de reconnaître dans les textes anciens des maladies précises, le vocabulaire est trop différent et trop vague par rapport à un discours médical. D’après des textes citant judāam, il suggère la syphilis qui est également plausible, puisqu'on admet aujourd'hui que la maladie 
existait en Eurasie bien avant Christophe Colomb. Comme la syphilis a des symptômes cutanés multiples et trompeurs (ulcérations spectaculaires, pour cela on l'appelait aussi la Grande Vérole), elle peut être compatible avec ces descriptions anciennes (in litt.. 4-11-2021). Le corps noir peut être, lui, compris dans un sens métaphorique.

33. Cette partie du texte est intéressante car cette " duperie ou tromperie » devait être assez fréquente, le musc étant un parfum fort et très apprécié des cultures arabes. C'est le produit d'un chevrotin porte-musc, Moschus moschiferus, qui vit au Tibet et en Sibérie, dans les zones froides d'Asie Centrale. Il faut tuer l'animal pour prendre cette petite poche qui se trouve près des glandes anales. Ce parfum était luxueux et donc cher et donc falsifié. L'animal est aujourd'hui prêt de disparaître, s'il ne l'est déjà. Cette falsification se pratiquait en Inde probablement où l'on avait à disposition les fruits de teck.

34. Voir note 8 plus haut sur l'importance de ce canal.

35. Ce cadi, né à Kūfa en 735, était célèbre pour son savoir de la jurisprudence islamiste. Il était un fqih hanifite, l'une des quatre écoles juridiques de l'Islam; celle-ci prévaut en Turquie.

36. C'est très vraisemblablement la presqu'île de Malaisie.

37. Ce serait plutôt un seul tronc, pour une pirogue, mais il reprend le terme planche pour en donner la taille, très longue et très large.

38. Cependant le dictionnaire de Monnier-William donne aussi varàdâtu Bhpr, et varàdâru Npr (p. 922).

39. C'est le nom d'un ancien royaume, au centre-est de l'Inde et bordant le golfe du Bengale.

40. Bontius (1582-1631), médecin et "pionnier en médecine tropicale d'origine hollandaise » (note 55 au texte de Cuvier (1769-1832), 2019 -, History of the Natural sciences, 2. European Travelers and the Early Dutch Naturalists. En ligne),,, mourut aux Indes et c'est Guillaume Pison, de Leyden qui publia son œuvre : Histoire naturelle des Indes orientales. « Il y cite (livre VI, 157) sous le nom de Kiati, et sous celui de Quercus indica, un arbre qu'il assimile au Chêne, à cause de la dureté de son bois qui le rend propre aux constructions durables... Rheede paraît croire que ce pourrait être le theka de Malabar, et observant que le mot iati signifie chêne dans l'Inde, il croît que le vrai nom doit être kayo iati, «bois de chêne ». Au reste la figure de Bontius ne répond nullement à celle du theka donné par Rheede.... Et on peut douter de l'identité de ces deux arbres, quoique adoptée par Adanson » (Dictionnaire des sciences naturelles... 1822, tome 24, p. 422 sous le nom de Kiati).

\section{RÉSUMÉS}

Le teck, Tectona grandis L.f., est connu depuis au moins les premiers siècles de notre ère. Il a semble-t-il été utilisé d'abord pour ses propriétés comme bois de construction marine. Ce sont les Persans, puis les Arabes avant l'Islam, qui ont utilisé ce bois qu'ils avaient presque à volonté. IL était en effet abondant sur les côtes occidentales de l'Inde et donc bien à la portée du golfe Persique et du sud de la péninsule Arabique. Ils ont organisé son commerce pendant de nombreux siècles. Bașra, au fond du golfe Persique recevait les grumes entières. Dès les débuts de l'Islam (622), ce bois précieux a été aussi utilisé dans la construction des mosquées de la péninsule Arabique, puis dans celles des plus prestigieuses mosquées du monde islamique, sans oublier les palais arabes, et ensuite les maisons, comme à Sīrāf. Ce sont les arabes, voyageurs, géographes et religieux, qui nous apportent ces informations détaillées. Cet arbre est originaire du nord de l'Indochine (Birmanie, Thaïlande, Laos) et d'Inde d'où les prêtres de Vishnou 
l'introduisirent en Indonésie. Cette diffusion a donné lieu à des dénominations variées, jati en Indonésien, tekka, saka, sag en indien, sāg puis sāj en arabe. Aujourd'hui cet arbre aux multiples propriétés, dont la plus importante est celle de protéger le fer, est planté en reboisement partout dans les zones tropicales avec des usages beaucoup plus triviaux.

Teak, Tectona grandis L.f., has been known since the first centuries of our era, at least. It was first used as marine construction wood. The Persians, and later the Arabs, before Islam, used this wood which was abundant in the region. It came from the western coasts of India and was very easy to reach from the Persian Gulf and the south of the Arabian Peninsula. They organized its trade for many centuries. Whole logs of wood reached Bașra, at the far end of the Persian Gulf. The precious wood had also been used, from the beginning of Islam (622) to build also many mosques in the Arabian Peninsula, and later on even the most prestigious ones of the Islamic world, as well as the Arab palaces and then houses, like the ones in Sīrāf. Arab travelers, geographers and religious figures are the sources of detailed information. This tree came from northern Indochina (Burma, Thailand, Laos) and India; it was brought to Java by Vishnu priests. This circulation gave rise to various names, jati in Indonesian, tekka, saka, sag, in Indian and sāg and then sāj in Arab. Nowadays, this tree with so many properties - the most important one being to protect the iron - is being planted for reforestation purposes everywhere in the tropical zones, with much more common uses.

\section{INDEX}

Keywords : teak, Tectona grandis, India, Persians, Arabs, naval constructions, framework, designation, circulation

Mots-clés : teck, Tectona grandis, Inde, Persans, Arabes, constructions, marines, charpente, dénominations, diffusion

\section{AUTEUR}

\section{FRANÇOISE AUBAILE-SALLENAVE}

Chargée de recherche honoraire, UMR 7206 Muséum national d'Histoire naturelle 